Cancerolagia

\section{A QUIMIOTERAPIA ADJUVANTE PARA CÂNCER DE MAMA ENGORDA?}

Pacientes recebendo quimioterapia adjuvante por câncer de mama têm tendência a ganhar peso e esta conseqüência indesejável da quimioterapia é extremamente preocupante para mulheres que já terminaram seu tratamento quimioterápico. A magnitude do ganho ponderal durante o tratamento quimioterápico de mulheres com câncer de mama varia de 2,I a 5,9 kg e, em algumas séries, encontrou-se também uma correlação prognóstica adversa do ganho ponderal durante a quimioterapia e um menor intervalo livre de doença'. Em nosso meio, conduzimos um estudo retrospectivo com o objetivo de avaliar a variação de peso durante tratamento quimioterápico para pacientes portadoras de câncer de mama. Cento e seis pacientes consecutivas com câncer de mama foram avaliadas. No grupo em quimioterapia adjuvante e neoadjuvante, houve um ganho de peso médio de $0,91 \pm 1,19 \%(p<0,00001)$ do peso corpóreo por mês de tratamento. Não observamos correlação estatisticamente significante entre variação de peso e sobrevida livre de doença ou sobrevida global ${ }^{2}$.

O ganho ponderal verificado durante 0 tratamento quimioterápico destas mulheres é basicamente devido ao aumento da gordura e água corporais sem aumento concomitante de massa muscular (obesidade sarcopênica) $)^{3}$.

A causa do ganho ponderal durante a quimioterapia adjuvante em pacientes com câncer de mama é provavelmente multifatorial. Em um estudo conduzido por Demrak et al. ${ }^{3}$ com 53 mulheres submetidas a tratamento adjuvante para câncer de mama, constatou-se que as mulheres que ganharam peso tiveram uma atividade física significativamente menor sem alteração significativa da ingesta ou do metabolismo basal. Apontam-se ainda como causas do ganho ponderal durante a quimioterapia a labilidade emocional associada a estresse psicológico, o início da menopausa durante a QT, estar fazendo dieta nos últimos seis meses, além do uso simultâneo de ablação ovariana ou corticóides. Um outro fator que não pode ser esquecido seria a freqüente associação de hipotireoidismo com câncer de mama, que em nossa experiência está presente em 16\% das pacientes. Possíveis alternativas para evitar o ganho ponderal durante a quimioterapia em mulheres com câncer de mama incluem aconselhamento rotineiro com nutricionista e aderência a um programa de exercício físico ${ }^{4}$.

\section{Auro del Giglio}

Referências

I. Bastarrachea J, Hortobagyi GN, Smith TL, Kau SW, Buzdar AU. Obesity as an adverse prognostic factor for patients receiving adjuvant chemotherapy for breast cancer. Ann Intern Med 1994; I 20(I): 18-25.

2. Costa LJ, Varella PC, Del Giglio A. Weight changes during chemotherapy for breast cancer. Sao Paulo Med J. 2002; I 20(4): I 13-7. 3. Demark-Wahnefried W, Peterson BL, Winer EP, Marks L, Aziz N, Marcom PK, et al. Changes in weight, body composition, and factors influencing energy balance among premenopausal breast cancer patients receiving adjuvant chemotherapy. J Clin Oncol 200 I; 19(9):238 I-9. 4. Rock CL, Demark-Wahnefried W. Nutrition and survival after the diagnosis of breast cancer: a review of the evidence. I Clin Oncol 2002;20(15):3302-16. Review. Erratum in: I Clin Oncol 2002;20(I8):3939.

\section{Clínica Cinurgica \\ COLOSTOMIAS TEMPORÁRIAS: QUANDO FECHAR?}

Apesar de uma diminuição global nas últimas duas décadas do número de realizações de colostomias temporárias, este ainda é um procedimento de grande importância no arsenal de opções cirúrgicas de um cirurgião geral.

Não se encontra consenso em relação ao tempo de fechamento da colostomia. 0 período clássico de 8 a 12 semanas, encontrado na maioria das publicações, deve ser analisado com grande senso crítico.

A literatura atual identifica taxas de complicações extremamente variadas, com índices que vão de $9 \%$ até quase $50 \%$, nas cirurgias de decolostomias!.
Fatores inerentes ao próprio paciente, tais como idade, comorbidades associadas e uso crônico de medicações, exercem influência direta na morbidade dessas operações, assim como o motivo que levou à realização de uma colostomia 2 .

Desta forma, uma diverticulite aguda complicada, um tumor de cólon obstrutivo, uma lesão colônica por projétil de arma de fogo ou arma branca, ou ainda uma perfuração durante um exame endoscópico provocam, dependendo do paciente, respostas metabólicas e endócrinas variáveis, promovendo também efeitos diversos no processo de cicatrização das feridas ${ }^{3}$.

Com isso, quando se programam as cirurgias de restituição do trânsito intestinal, o mais importante é aguardar a superação do trauma cirúrgico anterior, que é peculiar de paciente para paciente.

Não se deve jamais indicar uma decolostomia sem certificar-se do estado clínico atual do doente, assim também como a condição em que se encontram os segmentos intestinais envolvidos, avaliados através de estudos contrastados e ou endoscópicos.

Outra consideração importante é que, do ponto de vista técnico, colostomias feitas sem cuidado rigoroso, desde o correto posicionamento da alça até o uso de técnica adequada de maturação, estão intimamente relacionadas com maiores índices de complicações, devido a dificuldades técnicas no momento da decolos tomia onde muitas vezes são necessárias colectomias segmentares.

Assim sendo, o mesmo cuidado e precaução tido na hora de fechar uma colostomia, deve ser seguido na hora de confeccioná-la.

\section{Osvaldo Antonio Prado Castro Rodrigo VincenzI Ettore ferrari franciulli Paulo Kassab Elias JiRjoss Ilias}

Referências

I. Biondo-Simões MLP, Brenner S, Lemos R, Duck D, Rey SD. Análise das complicações pós-operatórias em decolostomias. Acta Cir Bras 2000; I5(supl. 3):53-7. 
2. Wong RW, Rapapport WD, Witzke DB, Putnam CW, Hunter GC. Factors influencing the safety of colostomy closure in the eldery. J Surg Res 1994; 57(2):289-92.

3. Ghorra SG, Rzeczycki TP, Natarajan N, Pricolo VE. Colostomy closure: impact of preoperative risk factors on morbidity. Am Surg 1999; 65(3):266-9.

\section{Clínica Médica}

\section{COMO DEVE SER TRATADA} A HIPERTENSÃO ARTERIAL

\section{PULMONAR?}

O tratamento atual da hipertensão arterial pulmonar (HAP) inclui medidas gerais e a utilização de novas drogas. Há recomendações a serem seguidas com o objetivo de evitar ou retardar a piora da função ventri cular direita e eventos tromboembólicos. Orienta-se limitar a atividade física de acordo com a sintomatologia, evitar altitudes elevadas (acima de $800 \mathrm{~m}$ ) bem como cabines de avião não-pressurizadas, e monitorização cuidadosa de procedimentos invasivos. Para pacientes do sexo feminino, a gravidez está contra-indicada pelo alto risco de falência ventricular direita e óbito.

O tratamento convencional da HAP consiste na anticoagulação crônica, controle da disfunção ventricular direita, uso de oxigênio domiciliar e vasodilatadores. Apesar de não haver estudos clínicos controlados, há evidências de que pacientes anti coagulados têm maior sobrevida. Recomenda-se o uso de warfarin, mantendo-se o INR (International Normalized Ratio) entre 2,0 e 2,5. Para o controle da disfunção ventricular direita, a digoxina é a droga mais utilizada. Diuréticos como furosemida e espironolactona ajudam a controlar a congestão sistêmica, tendo-se o cuidado de não diminuir excessivamente a pré-carga ventricular direita e conseqüente piora do débito cardíaco. O uso crônico de oxigênio domiciliar é recomendado nos pacientes com pressão arterial de oxigênio abaixo de $60 \mathrm{mmHg}$.

Antes de iniciar o tratamento com vasodilatador, orienta-se realizar estudo hemodi- nâmico, com o teste agudo de vasodilatação pulmonar. $O$ vasodilatador de escolha é o óxido nítrico ( 10 ppm, durante 10 minutos). Considera-se resposta positiva a redução de no mínimo 20\% na resistência vascular pulmonar, com respectivo aumento no índice cardíaco, ou redução da pressão média arterial pulmonar de pelo menos $10 \mathrm{mmHg}$, sendo o valor final inferior a $40 \mathrm{mmHg}$. $\mathrm{O}$ uso de bloqueadores de canais de cálcio em altas doses melhora a sobrevida de pacientes que respondem adequadamente ao teste vasodilatador.

Além da prostaciclina intravenosa (Epoprostenol), padrão-ouro no tratamento da HAP, com efeitos vasodilatador, antiplaquetário e antiproliferativo, surgem outros análogos com vias diversas de administração: oral (Beraprost); subcutânea (Treprostinil); inalatória (lloprost). Bons resultados têm sido obtidos com o bosentan, inibidor dos receptores da endotelina-I, administrado por via oral, com relatos de melhora na capacidade física, nas variáveis hemo dinâmicas e na sobrevida. Estudos clínicos isolados demonstram que o sildenafil, um inibidor da enzima fosfodiesterase-5, meIhora a capacidade física e a qualidade de vida em pacientes com HAP. Está em fase final um estudo multicêntrico, duplo-cego e randomizado para determinar a eficácia dessa droga em HAP. Os resultados preliminares de estudos não controlados são promissores.

Alguns exemplos de drogas utilizadas no tratamento da HAP são: I) Bosentan: $62,5 \mathrm{mg}, 2 x$ ao dia (por 30 dias); $125 \mathrm{mg}, 2 \mathrm{x}$ ao dia, a seguir; 2) lloprost: 13 a 15 mg, 6 a 9x ao dia; 3) Sildenafil: 50mg, $3 x$ ao dia (sujeito a modificação). Existem vários critérios para se avaliar a resposta a um tratamento instituído. Os pacientes que após três meses de tratamento não atingem 380 metros no teste de seis minutos, são considerados graves, com prognóstico reservado.

Além do tratamento medicamentoso, a atriosseptostomia pode melhorar a sinto matologia em casos selecionados. Como recurso final naqueles pacientes com disfunção ventricular refratária ao tratamento medicamentoso há o transplante pulmonar ou cardiopulmonar, com morbi- mortalidades semelhantes. $\mathrm{O}$ advento de novas drogas que estão melhorando a qualidade de vida e sobrevida dos pacientes deverá retardar a decisão pelo transplante pulmonar.

Alessandra C. Barreto Antonio Augusto Lopes

Referência

Diretriz: diagnóstico, avaliação e terapêutica da hipertensão pulmonar. Arq Bras Cardiol 2003; 8I(supl 8).

\section{Ginecolagia \\ COMO INVESTIGAR AS ANAMOLIAS DOS DUCTOS MÜLLERIANOS?}

O ginecologista deve estar atento quando atende casos de amenorréia associada a dor pélvica recorrente ou dismenorréia na adolescência. O raciocínio clínico deve ser complementado com as possibilidades propedêuticas atuais.

$\mathrm{Na}$ anamnese deve ser indagado sobre ocorrência ou não da menarca e se a história da paciente associa-se a queixas de dores no baixo ventre tipo cólica, muitas vezes cíclicas $\mathrm{e}$, não raro, progressivas. $\mathrm{O}$ exame ginecológico pode revelar hímen imperfurado, malformação congênita do trato genital feminino, onde ocorre falha de tunelização da membrana himenal; percebe-se, por vezes, septos vaginais.

Exames complementares como ultrasom, ressonância magnética, vaginoscopia, histeroscopia e videolaparoscopia devem ser obrigatórios no diagnóstico correto, além de assumir especial importância no planejamento cirúrgico. O ultra-som pode sugerir imagens de hidrossalpinge e/ou hematometra, associadas ou não a hidro ou hematocolpo. A ressonância magnética avalia imagens específicas do trato genital e é fundamental para mostrar os tipos de anomalias dos ductos müllerianos. A vaginoscopia é útil na caracterização da cavidade vaginal, tanto para diagnóstico de septos transversais ou longitudinais, quanto mensuração da profundidade vaginal. A histeroscopia diagnóstica 